\title{
Mesenchymal stem cells-derived exosomal microRNAs contribute to wound inflammation
}

\author{
Dongdong Ti, Haojie Hao, Xiaobing $\mathrm{Fu}^{*} \&$ Weidong Han ${ }^{* *}$ \\ Institute of Basic Medicine, College of Life Sciences, Chinese PLA General Hospital, Beijing 100853, China
}

Received August 25,2016; accepted October 16, 2016; published online November 18, 2016

\begin{abstract}
Clinical and experimental studies have highlighted the significance of inflammation in coordinating wound repair and regeneration. However, it remains challenging to control the inflammatory response and tolerance at systemic levels without causing toxicity to injured tissues. Mesenchymal stem cells (MSCs) possess potent immunomodulatory properties and facilitate tissue repair by releasing exosomes, which generate a suitable microenvironment for inflammatory resolution. Exosomes contain several effective bioactive molecules and act as a cell-cell communication vehicle to influence cellular activities in recipient cells. During this process, the horizontal transfer of exosomal microRNAs (miRNAs) to acceptor cells, where they regulate target gene expression, is of particular interest for understanding the basic biology of inflammation ablation, tissue homeostasis, and development of therapeutic approaches. In this review, we describe a signature of three specific miRNAs (miR-21, miR-146a, and miR-181) present in human umbilical cord MSC-derived exosomes (MSC-EXO) identified microarray chip analysis and focus on the inflammatory regulatory functions of these immune-related miRNAs. We also discuss the potential mechanisms contributing to the resolution of wound inflammation and tissue healing.
\end{abstract}

microRNA, inflammation, mesenchymal stem cell, exosome

Citation: Ti, D., Hao, H., Fu, X., and Han, W. (2016). Mesenchymal stem cells-derived exosomal microRNAs contribute to wound inflammation. Sci China Life Sci 59, 1305-1312. doi: 10.1007/s11427-016-0240-4

\section{INTRODUCTION}

Inflammation is an evolutionarily biophysical self-defense response of the body to harmful stimuli and aims to maintain a homeostatic state (Kizil et al., 2015). Immune cells, mainly resident macrophages, dendritic cells, and neutrophils, are activated during inflammation and recruited to the impaired site to initiate the healing process by eradicating pathogens and damaged cells. Although an acute and well-regulated inflammatory response is typically crucial for returning cells and organisms to homeostasis, chronic and inappropriate inflammation is hazardous to tissues and predisposes patients to the

*Corresponding author (email: fuxiaobing@vip.sina.com)

**Corresponding author (email: hanwdrsw69@yahoo.com) onset or progression of delayed wound healing (Sugimoto et al., 2016; Landén et al., 2016). Therefore, the regulation of inflammation is a potential therapeutic target for intervention to reduce the risk of disease and disability.

Recently, stem cell-based technologies using mesenchymal stem cells (MSCs) have been proposed for the treatment of inflammation-related diseases (Liu et al., 2016; Gao et al., 2016; Shen et al., 2015). MSCs are a population of self-renewing undifferentiated adult cells that can be obtained from different tissues (bone marrow, umbilical cord, adipose, etc.) and are typically defined as plastic-adherent, fibroblast-like cells with the potential to differentiate along a distinct variety of cell lineages. They can home to the injured sites, interact with several cells of the immune system, and modulate 
the local microenvironment through their unique immunological properties. Bury et al. found that MSC grafts markedly reduced the presence of pro-inflammatory macrophages and neutrophils, attenuated the innate inflammatory response, and facilitated bladder tissue regeneration (Bury et al., 2015). In addition, administered MSCs may inhibit dendritic cell differentiation and maturation, reduce lymphocyte activities, and decrease the cytotoxic activity of natural killer cells in acute kidney injury (De Miguel et al., 2012).

Increasing evidence has suggested that MSCs secrete exosomes as vital extracellular communicators to modulate the proliferation, activation, and effector function of targeted cells and have therapeutic effects on wound inflammation (Fontaine et al., 2016; Ophelders et al., 2016; Nakano et al., 2016). Exosomes, which were first defined by Trams et al., are small biological lipid membrane vesicles with diameters for 40-100 nm (Trams et al., 1981). Researchers determined the exosome biogenesis process in different cell types and successfully isolated these membranous bodies from diverse sources, ranging from bodily fluids to the supernatants of cultured cells. Under physiological and pathological conditions, these vesicles are formed by inward budding of late endosomes and then released into the extracellular environment upon fusion with the plasma membrane (Basso and Bonetto, 2016). They can shuttle biologically active molecules, such as intact and functional microRNAs (miRNAs), messenger RNAs (mRNAs), and proteins, to targeted recipient cells and reprogram cell behaviors (Zhou et al., 2016; Fang et al., 2016).

miRNAs are highly conserved, small (19-24 nt), non-coding RNA molecules that regulate gene expression at the post-transcriptional level by promoting the degradation or blocking of mRNA translation. It has been reported that several miRNAs play crucial roles in regulating different inflammatory responses in many tissues and affect the phenotype and development of immune cells. Dysregulation of miR-21 or miR-181 induces a chronic inflammatory state; this miRNA is key in controlling the balance between initial pro-inflammatory and later immuno-regulatory, anti-inflammatory responses (Garo and Murugaiyan, 2016; Sun et al., 2014). As master molecular switches, miRNAs exchange bio-information between neighboring cells and are important in exosome-mediated microenvironmental changes (Cavalieri et al., 2016; Navakanitworakul et al., 2016). Montecalvo et al. showed that miRNAs in exosomes are remarkably stable, likely because of their unique structure that protects them against harsh degrading conditions, such as low acidic $\mathrm{pH}$ and RNase-mediated degradation (Montecalvo et al., 2012). miRNAs transferred through exosomes, such as miR-146a and miR-155, are expressed in a tissue- and cell type-specific manner and can alter the host cell to evade the host's innate immune response and influence their survival and activated or differentiated status
(Alexander et al., 2015). In addition, the miRNA component of exosomes is heterogeneous and depends on the cells of origin. Various microenvironmental factors, including calcium concentration and growth factors, can also influence the transport of information by exosomes and their regulatory properties (Taverna et al., 2016).

MSC-derived exosomes (MSC-EXO) have been proposed as an ideal therapeutic strategy for wound inflammation. When exposed to an extremely harsh inflammatory microenvironment within injured sites, engrafted stem cells exhibit low survival rates, limiting their sustained reparative capacity. Exosomes resist external degradation and provide target cells with effective genetic information, and thus MSC-EXO may be non-invasive and dynamic candidates as gene therapy vectors to communicate important regulatory signals between cells and mediate protective signals against stress in other cells during wound inflammation. miRNA "marker signatures", which are carried and secreted by MSC-EXO, may participate in the resolution of chronic inflammation and enhance the curative effects of MSCs. A better understanding of the miRNA expression profiles and putative specific miRNA functions within MSC-EXO will facilitate further development of MSC-mediated exosome therapy aimed at the modification of wound inflammation.

\section{HOW MSCS FUNCTION IN WOUND INFLAMMATION}

Inflammation is not only an essential step of wound healing but also has large impacts on the final wound outcome. If unchecked and persistent, it can lead to delayed wounds that are characteristic of fibrosis, excessive scar formation, or inhibiting reepithelialization. Numerous basic and clinical data over the last decade indicate that the anti-inflammatory and immunomodulative properties of MSCs are particularly beneficial for poor wound treatment, as they advance the wound's capacity to progress beyond the inflammatory stage and not transit to an impaired wound state (Gao et al., 2016; van den Akker et al., 2013). After the immunomodulatory capacities are activated by the inflammatory microenvironment of the wound sites, MSCs secrete different bioactive components, exert pleiotropic effects on immune cells at injured sites, and play a protective role in remedying tissue inflammation and improving healing. In a mouse model of autoimmune encephalomyelitis, MSCs effectively inhibited Th17 cell polarization and ameliorated the development of autoimmune responses by secreting large amounts of soluble TNF receptor to antagonize TNF- $\alpha$ function (Ke et al., 2016). TNF- $\alpha$ is considered as a major proinflammatory medium in the promotion of Th17 cell differentiation and the pathophysiology of autoimmune encephalomyelitis. Additionally, Xu et al. found that accelerated diabetic wound healing by MSC treatment was associated with significantly up-regulated ex- 
pression of miR-146a and suppression of proinflammatory target genes expression (Xu et al., 2012). Based on these studies, MSC may reprogram a well-orchestrated and proregenerative microenvironment for inflammation adjustment then induce functional restoration of injured tissues, which may be primarily related to the paracrine mechanism triggered by MSCs. As active ingredients of trophic secretion by MSCs, exosomes are important regulators of cell-to-cell communication for inflammatory signaling and wound repair by shuttling functional materials such as protein and miRNAs. Studies have shown that MSC-EXO significantly depressed lymphocyte proliferation, restrained the inflammatory response, and played a cardioprotective role. A decrease in the concentration of exosomes, results in the slower recovery of damaged hearts (Teng et al., 2015). Thus, further investigations are necessary to explain the transporter roles of MSC-EXO in wound healing and analyze the composition of exosomes to reveal their detailed mechanisms in chronic inflammation.

\section{MIRNA EXPRESSION PATTERNS RELEASED BY MSC-EXO}

Computational and indirect evidence has indicated that miRNAs directly manage up to one-third of protein-coding genes. They act as critical immune-modulators of diverse processes, including the differentiation and induction of immune cells and maintenance of cellular homeostasis and functions (Mehta and Baltimore, 2016). For example, preconditioning of MSCs triggers internal changes in cells, possibly at the post-transcriptional and/or epigenetic levels, which are responsible for subsequent gene reprogramming or robust immune tolerance (Zhang et al., 2016; Dong et al., 2015; Aktas et al., 2016). In our previous study, we found that lipopolysaccharide (LPS) priming can augment the paracrine protective ability and therapeutic potentiality of MSCs; additionally, let-7b was distinctively shuttled by LPS-pretreated MSC-derived exosomes (LPS pre-Exo), may function in the balance of macrophage polarization to cure chronic inflammation and enhance skin wound repair (Ti et al., 2015). MiRNAs are induced in a time-specific manner, and the resulting regulatory network is often very extensive and complicated. Therefore, it is not surprising that miRNAs are partially taken up by MSCs as cargo in exosomes to influence coordinated changes in gene expression occurring during the resolution of wound inflammation.

Therefore, in order to explore whether MSC-EXO exert the immunomodulative properties through specific miRNAs, we cultured human umbilical cord MSCs at the 4th-5th passages, harvested exosomes from the supernatants of MSCs by gradient centrifugation, and conducted microarray analysis to determine the miRNA expression profile of MSC-EXO (Ti et al., 2015). Following probe screening and data normalization, we found 42 significantly differentially expressed
miRNAs compared to in human fibroblast-derived exosomes (FIB-EXO, Figure 1). Among 15 up-regulated miRNAs, three (miR-21, miR-146a, and miR-181) displayed the most significant and highest expression in MSC-EXO (data not shown). Next, we performed bioinformatics prediction to determine potential downstream targets of miR-21, miR-146a, and miR-181. According to the Reactome Database, targets of these three specific miRNAs were statistically enriched in genes and proteins participating in cellular development and function, innate immune response, inflammation, and cell signaling. Particularly, the Toll-like receptor (TLR) pathway and IL-6 pathway have been shown to be important cellular pathways fundamentally required for activation of the immune system and orchestration of the inflammatory process after tissue damage.

Given the essential role of the miRNA processing machinery in immune cell development and inflammatory responses, we hypothesized that these three specific miRNAs in MSCEXO could modulate the expression of inflammation-related genes, which may reduce delayed inflammation and enhance wound healing. However, little is known about how and to what extent these miRNAs function in the immune stimulatory program of MSCs, which is an important issue for potential therapeutic molecule discovery to coordinate uncontrolled inflammation. We reviewed their biological activities interacting with inflammatory cells and directly regulating intracellular regulatory mechanisms or affecting the cellular phenotype in the resolution of wound inflammation.

\section{miR-21}

miR-21 can be selectively packaged from original cells into exosomes and acts as a rheostat to modulate immune cell function and the inflammatory response. Pieters et al. demonstrated that extracellular vesicles, including exosomes, which were isolated from commercial bovine milk, could induce Th17 differentiation in murine splenocytes, and miR-21 was present in the immunoregulatory cargo carried by these extracellular vesicles (Pieters et al., 2015). In addition, data analyses of the human MSC secretome confirmed that miR-21 protects MSCs under serum-deprived conditions and can trigger key biological functions in recipient cells (Nie et al., 2011). Efficient efferocytosis induces potent anti-inflammatory and immunosuppressive mechanisms that are required for the timely resolution of inflammation. Notably, miR-21 is efferocytosis-inducible in macrophages, subsequently promoting efferocytosis progression. In the regulatory loop, miR-21 silences PTEN and GSK3 $\beta$, dampens NFאB activation, and promotes c-Jun/AP1 activities, which control the inflammatory response and promote wound healing (Das et al., 2014). Furthermore, miR-21 is instrumental in the regulation of macrophage phenotype and inflammation ablation for efficient stem cell-dependent tissue repair. In response to bacterial infections, miR-21 


\section{FIB-EXO1 FIB-EXO2 FIB-EXO3 MSC-EXO1 MSC-EXO2 MSC-EXO3}

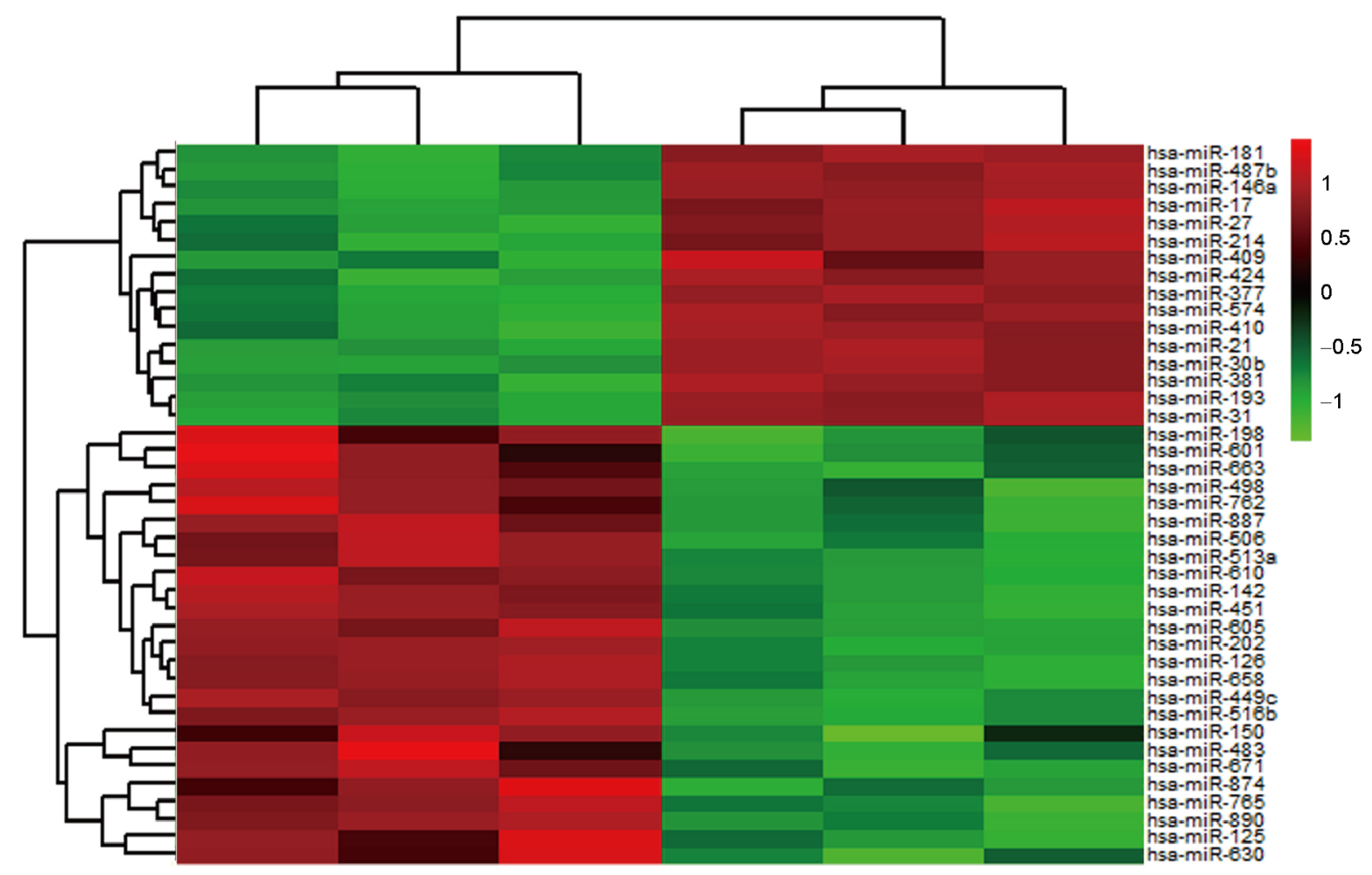

Figure 1 Analysis of miRNA expression profiling in MSC-EXO. A heatmap was generated after supervised hierarchical cluster analysis. Differential miRNA expression is shown in red (upregulation) versus green (downregulation) intensity (MSC-EXO versus FIB-EXO, 2-fold change, $P<0.05$ ).

leads to NFKB activation in pro-inflammatory macrophages, thus promoting both inflammation and the expression of antiinflammatory genes. It then depresses PTEN in anti-inflammatory macrophages, resulting in AKT activation to cause inflammation alleviation and wound healing (Perdiguero et al., 2011). Hence, we suggest that miR-21 is a highly interesting and significant player in downstream inflammatory pathways and post-host immune responses mediated by diverse immune cells; the expression of miR-21 in MSC-EXO may be involved in the modulation of the inflammatory response during tissue repair.

\section{miR-146a}

miR-146a was previously shown to be highly expressed in multiple types of adult stem cells. Furthermore, miR-146a may play a role in mediating short-term memory in immune system cells and MSCs after environmental stimulation. When MSCs migrate to the inflammatory microenvironment in injured tissues, they possess a "short-term memory" for danger signals and display enhanced therapeutic immunoregulation efficacy by inducing stimulus-responsive regulatory molecules, such as miR-146a (Liu et al., 2016). Saba et al. suggested that in response to inflammatory cues, miR-146a can function as a key regulator of the innate immune response to arrest the expression of several pro-inflammatory factors, including TNF- $\alpha$, IL- 6 , and IFN- $\gamma$, and direct the in- flammatory response (Saba et al., 2014). miR-146a can also protect the functional capabilities and longevity of murine hematopoietic stem cells against prolonged inflammation; these cells are critical for an adequate inflammatory and immune response in healthy individuals (Zhao et al., 2013). In addition, miR-146a regulates not only the acute $\mathrm{T}$ cell response but also chronic $\mathrm{T}$ cell hyper-inflammatory response and development of $\mathrm{T}$ cell-associated autoimmunity. This is achieved by repressing NFKB activity and monitoring the T cell response to antigen stimulation during $\mathrm{T}$ cell activation (Meisgen et al., 2014). Etzrodt et al. showed that miR-146a may regulate monocyte subset population dynamics. In vivo enhancement of miR-146 transcription in macrophages and monocytes can attenuate monocyte/macrophage activation and inhibit pro-inflammatory macrophage responses to suppress NFкB-mediated inflammation (Etzrodt et al., 2012). Thus, MSC-shuttled exosomal miR-146a may have a diverse and critical role in limiting an excessive inflammatory reaction, and therefore represent a future therapeutic target for exogenous modulation of wound inflammation.

\section{miR-181}

Experiments have shown that miR-181 plays an important role in the development, differentiation, and activation of $\mathrm{T}$ lymphocytes, B lymphocytes, and natural killer cells (Chen et al., 2004; Beaulieu et al., 2013). The immune system 
mounts rapid and specific defense responses and develops and maintains a steady microenvironment; if deregulated, this can result in decreased regenerative capacity and various pathological disorders, including defects in host-pathogen interactions, autoimmune diseases, or chronic inflammation. Thus, miR-181 may provide excellent spatio-temporal monitoring of immune cells, modulate the strength and threshold of immune signals under physiological and pathological conditions, and switch outcomes on immune responses for tissue homeostasis. When miR-181 is deficient in a specific tissue in mice, conventional $\mathrm{T}$ cell and natural killer $\mathrm{T}$ cell development were perturbed and PI3K signaling was impaired, which results from the cumulative effect of increased PTEN expression (Henao-Mejia et al., 2013). Dan et al. suggested that miR-181 upregulation is the primary host protective method against endotoxin shock, as it can switch the immune status from hyperinflammation to endotoxin tolerance by quickly decreasing inflammatory cytokine expression without influencing the expression of anti-inflammatory cytokines (Dan et al., 2014). In an experimental neuroinflammatory model, miR-181 was found to be necessary for the reactive phenotype of astrocytes to inflammatory settings by reprogramming the transcriptional profiles of general cell differentiation and stress responses (Hutchison et al., 2013). These results clearly illustrate that miR-181 plays a central role in anabolic metabolism in immune cells and fine-tunes their immunological functions to prevent overwhelming inflammation during tissue regeneration.

\section{UNIQUE MICRORNA EXPRESSION IN MSC-EXO CONTRIBUTES TO THE RESOLUTION OF WOUND INFLAMMATION}

Increasing data have shown that MSC-EXO have specific biological functions for modulating the immune and inflammatory response and repairing tissue damage. The manipulation of genetic elements using selective exosomal regulation may have fully or partially penetrant effects on pathological conditions at the cellular and systemic levels. Blazquez et al. showed that MSC-EXO have an inhibitory role in the differentiation, proliferation, and activation of T cells and IFN- $\gamma$ release during the treatment of inflammation-related diseases (Blazquez et al., 2014). These observations suggest that specific therapeutic miRNA populations in MSC-EXO reshape the immunomodulatory strength of MSCs to mediate target immune cell activation, phenotypic modification, and reprogramming of cell function. Moreover, in our study, bioinformatic approaches indicated that specific miRNAs shuttled by MSC-EXO have complex pleiotropic effects on many aspects of inflammation. These miRNAs are predicted to target immune and inflammatory response genes, including transcription factors, co-factors, and chromatin modifiers. In addition, critical proteins involved in regulating inflammation, such as
TLRs and IL-6, have been suggested to act as links between MSC-EXO and inflammatory pathway activation in immune cells. Therefore, studies of the immune signals and mechanistic pathways regulated by miR-21, miR-146a, and miR-181 may enable better modulation of inflammation and advanced methods for stem cell-mediated tissue repair.

\section{TLR signaling pathway}

TLRs are a class of membrane-bound pattern recognition receptors that are responsible for recognizing particular pathogen-associated molecular patterns from infectious pathogens. The immune system mainly detects and identifies pathogenic molecules via TLRs then triggers various transcription factors, leading to strong production of pro-inflammatory cytokines (Sabouri et al., 2014; Helley et al., 2015). Inappropriate quantities or qualities of TLR ligands or aberrant response to TLR activation have been observed in various impaired inflammatory conditions. miRNA, which inhibits protein expression through mRNA degradation or translational repression, can be used to precisely regulate the immune system and immune cell activity. Therefore, specific miRNAs in MSC-EXO may strictly modulate TLR-signaling pathways to prevent excessive inflammation and achieve a balanced microenvironment. For example, miR-146a is a regulatory element in keratinocyte innate immunity that suppresses the expression of a large number of inflammatory genes under homeostatic conditions and serves as a potent negative feedback regulator of the TLR proinflammatory pathway. Furthermore, miR-181 directly binds to the 3' untranslated region of IL-8, and represses its expression in human fibroblasts. Thus, upregulation of miR-181 may be associated with the homeostatic response to inflammatory stimuli such as IL- 8 by TLR pathway activation (Galicia et al., 2014).

Once activated, the TLR pathway acts through different intracellular signaling cascades and leads to activation of $\mathrm{NF \kappa B}$ and TRIF-dependent signaling, resulting in cytokine, chemokine, and cell adhesion molecule induction. Evidence is accumulating that miRNAs function as important immunomodulators to fine-tune the TLR-induced NFKB signaling pathways and downstream consequences. miR-21 was induced by the TLR/NFKB pathways in LPS priming human peripheral blood mononuclear cells, in turn, repressing the NFKB pathway and upregulating IL-10 production (Sheedy et al., 2010). Feng et al. found that miR-21 inhibits lipid-laden foam cell formation and inflammatory responses in LPS-stimulated macrophages through the TLR4-NF- $\kappa B$ pathway (Feng et al., 2014). Hence, we hypothesize that particular miRNAs in MSC-EXO create a regulatory feedback to monitor certain TLR-activated transcription factor-dependent pathways and timely decrease and/or terminate TLR signaling by targeting the expression of critical signaling components. 


\section{IL-6 signaling pathway}

IL-6 is a pleiotropic cytokine that regulates multiple biological processes, including acute phase responses, cell regeneration, inflammation, and immune responses. As a pro-inflammatory soluble factor, IL-6 produced by monocytes-macrophages represents a key molecule in the induction and maintenance of delayed inflammation during RA progression. Some evidence suggests that IL-6 compromises tissue repair by shifting acute inflammation into a more chronic profibrotic state induced by Th1 cells as a consequence of recurrent inflammation (Fielding et al., 2014). In all cases tested, blockade of IL- 6 by specific miRNAs in MSC-EXO may be a useful therapeutic measure for wound inflammation. For example, in a mathematical inflammation model, miR-146 and miR-21 significantly controlled the coupled inflammatory reactions by fine-tuning the activation dynamics of the key mediators of IL-6 (Xue et al., 2013). After LPS exposure, increased miR-181 may be involved in decreasing IL-6 expression, which may be also an important regulatory mechanism for controlling the pro-inflammatory immune response and induction of endotoxin tolerance (Zhang et al., 2015).

In the classic signaling pathway, IL-6 binding to the membrane-bound IL-6 receptor (IL-6R) is followed by homodimerization of IL-6R with its signal-transducing element gp130 and initiation of the phosphorylation of the transcription factors STAT3. It has been demonstrated that the IL-6-gp130-STAT3 signaling axis is necessary for the orchestration of inflammatory processes in animal models (Hirano, 2010). The identification of novel upstream modulators or downstream targets of the IL-6/STAT3 pathway will provide insight into the involvement of altered miRNA expression in the progression of chronic inflammation. In RA patients, the expression levels of miR-21 were significantly lower and accompanied by increased STAT3 expression and activation (Dong et al., 2014). Moreover, Iliopoulos et al. found that miR-21 and miR-181 are rapidly and dramatically regulated during cellular transformation. A transient inflammatory signal triggers the inflammatory feedback loop involving IL-6, STAT3, miR-21, and miR-181 and induces an epigenetic switch that links inflammation to cancer; the transformed state can be interrupted by any genetic or environmental change affecting the factors of the inflammatory feedback loop (Iliopoulos et al., 2010). These complex mechanisms provide a paradigm for molecular target therapy in which a key process of disease progression involves miRNA-induced changes in response to an inflammatory signal.

\section{PERSPECTIVES AND FINAL REMARKS}

In this review, we examined the miRNAs profiling in MSC-EXO and explored the impacts of selected miRNAs
(miR-21, miR-146a, and miR-181) on inflammatory responses and tolerance at the molecular, cellular, and systemic levels. miRNA expression may be turned on or off at the transcriptional level upon ligand stimulation and can have either positive or negative effects on the expression of signaling components that impact the development or function of different target cells. In some inflammatory disorders, studies have suggested that prominent up-regulation of miRNAs, such as miR21, is specifically involved in modulating TLR associated NFKB signaling networks activated by the pathological microenvironment (Kalla et al., 2015). Conceivably, miR146a is differentially expressed in various autoimmune diseases including rheumatoid arthritis and multiple sclerosis and its regulation may influence the development or prevention of autoimmunity (Dai and Ahmed, 2011). As exosomes naturally transfer RNA molecules between cells, this property may be useful in gene therapy, in which a vector is used to deliver therapeutic nucleic acids to the patient's target cells. Researchers have reported that exosomes can deliver synthetic miR-143 and are a potentially efficient and functional delivery system (Shimbo et al., 2014). These examples suggest that MSC-EXO may balance the dysregulated inflammatory condition without causing significant toxicity through specific miRNAs and can be used as efficient vehicles in RNA-based therapeutic strategies. Such efforts will ultimately reveal the immunoregulation function of miRNA and evaluation of whether their release via MSC-EXO is involved in the resolution of chronic inflammation and orchestrating wound healing.

Compliance and ethics The author(s) declare that they have no conflict of interest.

Acknowledgements This work was supported by the National Basic Science and Development Program (2012CB518103, 2012CB518105), National High Technology Research and Development Program of China (2013AA020105, 2012AA020502), and National Natural Science Foundation of China (81121004, 81501682, 81230041).

Aktas, E., Chamberlain, C.S., Saether, E.E., Duenwald-Kuehl, S.E., Kondratko-Mittnacht, J., Stitgen, M., Lee, J.S., Clements, A.E., Murphy, W.L., and Vanderby, R. (2016). Immune modulation with primed mesenchymal stem cells delivered via biodegradable scaffold to repair an Achilles tendon segmental defect. J Orthop Res doi: 10.1002/jor.23258.

Alexander, M., Hu, R., Runtsch, M.C., Kagele, D.A., Mosbruger, T.L., Tolmachova, T., Seabra, M.C., Round, J.L., Ward, D.M., and O'Connell, R.M. (2015). Exosome-delivered microRNAs modulate the inflammatory response to endotoxin. Nat Commun 6, 7321.

Basso, M., and Bonetto, V. (2016). Extracellular vesicles and a novel form of communication in the brain. Front Neurosci 10, 127.

Beaulieu, A.M., Bezman, N.A., Lee, J.E., Matloubian, M., Sun, J.C., and Lanier, L.L. (2013). MicroRNA function in NK-cell biology. Immunol Rev 253, 40-52.

Blazquez, R., Sanchez-Margallo, F.M., de la Rosa, O., Dalemans, W., Alvarez, V., Tarazona, R., and Casado, J.G. (2014). Immunomodulatory potential of human adipose mesenchymal stem cells derived exosomes 
on in vitro stimulated T cells. Front Immunol 5, 556.

Bury, M.I., Fuller, N.J., Wethekam, L., and Sharma, A.K. (2015). Bone marrow derived cells facilitate urinary bladder regeneration by attenuating tissue inflammatory responses. Cent European J Urol 68, 115-120.

Cavalieri, D., Rizzetto, L., Tocci, N., Rivero, D., Asquini, E., Si-Ammour, A., Bonechi, E., Ballerini, C., and Viola, R. (2016). Plant microRNAs as novel immunomodulatory agents. Sci Rep 6, 25761.

Chen, C.Z., Li, L., Lodish, H.F., and Bartel, D.P. (2004). MicroRNAs modulate hematopoietic lineage differentiation. Science 303, 83-86.

Dai, R., and Ahmed, S.A. (2011). MicroRNA, a new paradigm for understanding immunoregulation, inflammation, and autoimmune diseases. Transl Res 157, 163-179.

Dan, C., Jinjun, B., Zi-Chun, H., Lin, M., Wei, C., Xu, Z., Ri, Z., Shun, C., Wen-Zhu, S., Qing-Cai, J., and Wu, Y. (2014). Modulation of TNF- $\alpha$ mRNA stability by human antigen R and miR181s in sepsis-induced immunoparalysis. EMBO Mol Med 7, 140-157.

Das, A., Ganesh, K., Khanna, S., Sen, C.K., and Roy, S. (2014). Engulfment of apoptotic cells by macrophages: a role of microRNA-21 in the resolution of wound inflammation. J Immunol 192, 1120-1129.

P. De Miguel, M., Fuentes-Julian, S., Blazquez-Martinez, A., Y. Pascual, C., A. Aller, M., Arias, J., and Arnalich-Montiel, F. (2012). Immunosuppressive properties of mesenchymal stem cells: advances and applications. CMM 12, 574-591.

Dong, L., Hao, H.J., Han, W.D., and Fu, X.B. (2015). The role of the microenvironment on the fate of adult stem cells. Sci China Life Sci 58, 639-648.

Dong, L., Wang, X., Tan, J., Li, H., Qian, W., Chen, J., Chen, Q., Wang, J., Xu, W., Tao, C., and Wang, S. (2014). Decreased expression of microRNA-21 correlates with the imbalance of Th17 and Treg cells in patients with rheumatoid arthritis. J Cell Mol Med 18, 2213-2224.

Etzrodt, M., Cortez-Retamozo, V., Newton, A., Zhao, J., Ng, A., Wildgruber, M., Romero, P., Wurdinger, T., Xavier, R., Geissmann, F., Meylan, E., Nahrendorf, M., Swirski, F.K., Baltimore, D., Weissleder, R., and Pittet, M.J. (2012). Regulation of monocyte functional heterogeneity by miR146a and Relb. Cell Rep 1, 317-324.

Fang, S., Xu, C., Zhang, Y., Xue, C., Yang, C., Bi, H., Qian, X., Wu, M., Ji, K., Zhao, Y., Wang, Y., Liu, H., and Xing, X. (2016). Umbilical cord-derived mesenchymal stem cell-derived exosomal micrornas suppress myofibroblast differentiation by inhibiting the transforming growth factor- $\beta /$ SMAD2 pathway during wound healing. Stem Cells Transl Med $5,1425-1439$.

Feng, J., Li, A., Deng, J., Yang, Y., Dang, L., Ye, Y., Li, Y., and Zhang, W. (2014). miR-21 attenuates lipopolysaccharide-induced lipid accumulation and inflammatory response: potential role in cerebrovascular disease. Lipids Health Dis 13, 27.

Fielding, C.A., Jones, G.W., McLoughlin, R.M., McLeod, L., Hammond, V.J., Uceda, J., Williams, A.S., Lambie, M., Foster, T.L., Liao, C.T., Rice, C.M., Greenhill, C.J., Colmont, C.S., Hams, E., Coles, B., Kift-Morgan, A., Newton, Z., Craig, K.J., Williams, J.D., Williams, G.T., Davies, S.J., Humphreys, I.R., O’Donnell, V.B., Taylor, P.R., Jenkins, B.J., Topley, N., and Jones, S.A. (2014). Interleukin-6 signaling drives fibrosis in unresolved inflammation. Immunity 40, 40-50.

Fontaine, M.J., Shih, H., Schäfer, R., and Pittenger, M.F. (2016). Unraveling the mesenchymal stromal cells' paracrine immunomodulatory effects. Transfus Med Rev 30, 37-43.

Galicia, J.C., Naqvi, A.R., Ko, C.C., Nares, S., and Khan, A.A. (2014). MiRNA-181a regulates Toll-like receptor agonist-induced inflammatory response in human fibroblasts. Genes Immun 15, 333-337.

Gao, F., Chiu, S.M., Motan, D.A.L., Zhang, Z., Chen, L., Ji, H.L., Tse, H.F., Fu, Q.L., and Lian, Q. (2016). Mesenchymal stem cells and immunomodulation: current status and future prospects. Cell Death Dis 7, e2062.

Garo, L.P., and Murugaiyan, G. (2016). Contribution of microRNAs to autoimmune diseases. Cell Mol Life Sci 73, 2041-2051.

Helley, M.P., Abate, W., Jackson, S.K., Bennett, J.H., and Thompson, S.W.N.
(2015). The expression of Toll-like receptor 4, 7 and co-receptors in neurochemical sub-populations of rat trigeminal ganglion sensory neurons. Neuroscience 310, 686-698.

Henao-Mejia, J., Williams, A., Goff, L.A., Staron, M., Licona-Limón, P., Kaech, S.M., Nakayama, M., Rinn, J.L., and Flavell, R.A. (2013). The microRNA miR-181 is a critical cellular metabolic rheostat essential for NKT cell ontogenesis and lymphocyte development and homeostasis. Immunity 38, 984-997.

Hirano, T. (2010). Interleukin 6 in autoimmune and inflammatory diseases: a personal memoir. Proc Jpn Acad Ser B 86, 717-730.

Hutchison, E.R., Kawamoto, E.M., Taub, D.D., Lal, A., Abdelmohsen, K., Zhang, Y., Wood Iii, W.H., Lehrmann, E., Camandola, S., Becker, K.G., Gorospe, M., and Mattson, M.P. (2013). Evidence for miR-181 involvement in neuroinflammatory responses of astrocytes. Glia 61, 1018-1028.

Iliopoulos, D., Jaeger, S.A., Hirsch, H.A., Bulyk, M.L., and Struhl, K. (2010). STAT3 activation of miR-21 and miR-181b-1 via PTEN and CYLD are part of the epigenetic switch linking inflammation to cancer. Mol Cell 39, 493-506.

Kalla, R., Ventham, N.T., Kennedy, N.A., Quintana, J.F., Nimmo, E.R., Buck, A.H., and Satsangi, J. (2015). MicroRNAs: new players in IBD. Gut 64, 504-513.

Ke, F., Zhang, L., Liu, Z., Yan, S., Xu, Z., Bai, J., Zhu, H., Lou, F., Cai, W., Sun, Y., Gao, Y., Wang, H., and Wang, H. (2016). Soluble tumor necrosis factor receptor 1 released by skin-derived mesenchymal stem cells is critical for inhibiting Th17 cell differentiation. Stem Cells Transl Med 5, 301-313.

Kizil, C., Kyritsis, N., and Brand, M. (2015). Effects of inflammation on stem cells: together they strive? EMBO Rep 16, 416-426.

Landén, N.X., Li, D., and Ståhle, M. (2016). Transition from inflammation to proliferation: a critical step during wound healing. Cell Mol Life Sci 73, 3861-3885.

Liu, G.Y., Liu, Y., Lu, Y., Qin, Y.R., Di, G.H., Lei, Y.H., Liu, H.X., Li, Y.Q., Wu, C., Hu, X.W., and Duan, H.F. (2016). Short-term memory of danger signals or environmental stimuli in mesenchymal stem cells: implications for therapeutic potential. Cell Mol Immunol 13, 369-378.

Liu, J., Han, Z., Han, Z., and He, Z. (2015). Mesenchymal stem cell-conditioned media suppresses inflammation-associated overproliferation of pulmonary artery smooth muscle cells in a rat model of pulmonary hypertension. Exp Ther Med 11, 467-475.

Mehta, A., and Baltimore, D. (2016). MicroRNAs as regulatory elements in immune system logic. Nat Rev Immunol 16, 279-294.

Meisgen, F., Xu Landén, N., Wang, A., Réthi, B., Bouez, C., Zuccolo, M., Gueniche, A., Ståhle, M., Sonkoly, E., Breton, L., and Pivarcsi, A. (2014). MiR-146a negatively regulates TLR2-induced inflammatory responses in keratinocytes. J Invest Dermatol 134, 1931-1940.

Montecalvo, A., Larregina, A.T., Shufesky, W.J., Beer Stolz, D., Sullivan, M.L.G., Karlsson, J.M., Baty, C.J., Gibson, G.A., Erdos, G., Wang, Z., Milosevic, J., Tkacheva, O.A., Divito, S.J., Jordan, R., Lyons-Weiler, J., Watkins, S.C., and Morelli, A.E. (2012). Mechanism of transfer of functional microRNAs between mouse dendritic cells via exosomes. Blood 119, 756-766.

Nakano, M., Nagaishi, K., Konari, N., Saito, Y., Chikenji, T., Mizue, Y., and Fujimiya, M. (2016). Bone marrow-derived mesenchymal stem cells improve diabetes-induced cognitive impairment by exosome transfer into damaged neurons and astrocytes. Sci Rep 6, 24805.

Navakanitworakul, R., Hung, W.T., Gunewardena, S., Davis, J.S., Chotigeat, W., and Christenson, L.K. (2016). Characterization and small RNA content of extracellular vesicles in follicular fluid of developing bovine antral follicles. Sci Rep 6, 25486.

Nie, Y., Han, B.M., Liu, X.B., Yang, J.J., Wang, F., Cong, X.F., and Chen, X. (2011). Identification of MicroRNAs involved in hypoxia- and serum deprivation-induced apoptosis in mesenchymal stem cells. Int J Biol Sci 7, 762-768.

Ophelders, D.R.M.G., Wolfs, T.G.A.M., Jellema, R.K., Zwanenburg, A., Andriessen, P., Delhaas, T., Ludwig, A.K., Radtke, S., Peters, V., 
Janssen, L., Giebel, B., and Kramer, B.W. (2016). Mesenchymal stromal cell-derived extracellular vesicles protect the fetal brain after hypoxia-ischemia. Stem Cells Transl Med 5, 754-763.

Perdiguero, E., Sousa-Victor, P., Ruiz-Bonilla, V., Jardí, M., Caelles, C., Serrano, A.L., and Muñoz-Cánoves, P. (2011). p38/MKP-1-regulated AKT coordinates macrophage transitions and resolution of inflammation during tissue repair. J Cell Biol 195, 307-322.

Pieters, B.C.H., Arntz, O.J., Bennink, M.B., Broeren, M.G.A., van Caam, A.P.M., Koenders, M.I., van Lent, P.L.E.M., van den Berg, W.B., de Vries, M., van der Kraan, P.M., and van de Loo, F.A.J. (2015). Commercial cow milk contains physically stable extracellular vesicles expressing immunoregulatory TGF- $\beta$. PLoS ONE 10, e0121123.

Saba, R., Sorensen, D.L., and Booth, S.A. (2014). MicroRNA-146a: a dominant, negative regulator of the innate immune response. Front Immunol 5,578 .

Sabouri, A.H., Marcondes, M.C.G., Flynn, C., Berger, M., Xiao, N., Fox, H.S., and Sarvetnick, N.E. (2014). TLR signaling controls lethal encephalitis in WNV-infected brain. Brain Res 1574, 84-95.

Sheedy, F.J., Palsson-McDermott, E., Hennessy, E.J., Martin, C., O'Leary, J.J., Ruan, Q., Johnson, D.S., Chen, Y., and O'Neill, L.A.J. (2010). Negative regulation of TLR4 via targeting of the proinflammatory tumor suppressor PDCD4 by the microRNA miR-21. Nat Immunol 11, 141-147.

Shen, Q., Zhang, L., Chai, B.F., and Ma, X. (2015). Isolation and characterization of mesenchymal stem-like cells from human nucleus pulposus tissue. Sci China Life Sci 58, 509-511.

Shimbo, K., Miyaki, S., Ishitobi, H., Kato, Y., Kubo, T., Shimose, S., and Ochi, M. (2014). Exosome-formed synthetic microRNA-143 is transferred to osteosarcoma cells and inhibits their migration. Biochem Biophys Res Commun 445, 381-387.

Sugimoto, M.A., Sousa, L.P., Pinho, V., Perretti, M., and Teixeira, M.M. (2016). Resolution of inflammation: what controls its onset? Front Immunol 7, 160.

Sun, X., Sit, A., and Feinberg, M.W. (2014). Role of miR-181 family in regulating vascular inflammation and immunity. Trends Cardiovasc Med 24, 105-112.

Taverna, S., Fontana, S., Monteleone, F., Pucci, M., Saieva, L., De Caro, V., Cardinale, V.G., Giallombardo, M., Vicario, E., Rolfo, C., Leo, G.D., and Alessandro, R. (2016). Curcumin modulates chronic myelogenous leukemia exosomes composition and affects angiogenic phenotype, via exosomal miR-21. Oncotarget 24, 30420.

Teng, X., Chen, L., Chen, W., Yang, J., Yang, Z., and Shen, Z. (2015). Mesenchymal stem cell-derived exosomes improve the microenvironment of infarcted myocardium contributing to angiogenesis and anti-inflammation. Cell Physiol Biochem 37, 2415-2424.

Ti, D., Hao, H., Tong, C., Liu, J., Dong, L., Zheng, J., Zhao, Y., Liu, H., Fu, X., and Han, W. (2015). LPS-preconditioned mesenchymal stromal cells modify macrophage polarization for resolution of chronic inflammation via exosome-shuttled let-7b. J Transl Med 13, 308.

Trams, E.G., Lauter, C.J., Norman Salem, J., and Heine, U. (1981). Exfoliation of membrane ecto-enzymes in the form of micro-vesicles. Biochim Biophys Acta 645, 63-70.

van den Akker, F., de Jager, S.C.A., and Sluijter, J.P.G. (2013). Mesenchymal stem cell therapy for cardiac inflammation: immunomodulatory properties and the influence of Toll-like receptors. Mediators Inflamm 2013, $1-13$.

Xu, J., Wu, W., Zhang, L., Dorset-Martin, W., Morris, M.W., Mitchell, M.E., and Liechty, K.W. (2012). The role of microRNA-146a in the pathogenesis of the diabetic wound-healing impairment: correction with mesenchymal stem cell treatment. Diabetes 61, 2906-2912.

Xue, X., Xia, W., and Wenzhong, H. (2013). A modeled dynamic regulatory network of NF- $\kappa$ B and IL-6 mediated by miRNA. Biosystems 114, 214-218.

Zhang, W., Shen, X., Xie, L., Chu, M., and Ma, Y. (2015). MicroRNA-181b regulates endotoxin tolerance by targeting IL-6 in macrophage RAW264.7 cells. J Inflamm 12, 18.

Zhang, Y., Lei, W., Yan, W., Li, X., Wang, X., Zhao, Z., Hui, J., Shen, Z., and Yang, J. (2016). microRNA-206 is involved in survival of hypoxia preconditioned mesenchymal stem cells through targeting Pim-1 kinase. Stem Cell Res Ther 7, 61.

Zhao, J.L., Rao, D.S., O’Connell, R.M., Garcia-Flores, Y., and Baltimore, D. (2013). MicroRNA-146a acts as a guardian of the quality and longevity of hematopoietic stem cells in mice. eLife 2, e00537.

Zhou, Y., Zhou, G., Tian, C., Jiang, W., Jin, L., Zhang, C., and Chen, X. (2016). Exosome-mediated small RNA delivery for gene therapy. WIREs RNA 7, 758-771.

Open Access This article is distributed under the terms of the Creative Commons Attribution License which permits any use, distribution, and reproduction in any medium, provided the original author(s) and source are credited. 